\title{
Sex Differences in Peripheral Augmentation Index and Arterial Reservoir Pressure during Upper Limb Postural Shifts
}

\author{
Kevin S. Heffernan, ${ }^{1}$ Ari G. Kasprowicz, ${ }^{1}$ Brendan J. Tarzia, ${ }^{1}$ \\ Justin E. Davies, ${ }^{2}$ and Darren P. Casey ${ }^{3}$ \\ ${ }^{1}$ The Human Performance Laboratory, Department of Exercise Science, Syracuse University, Syracuse, NY 13244, USA \\ ${ }^{2}$ National Heart and Lung Institute, International Centre for Circulatory Health, \\ Imperial College London \& Imperial College Healthcare NHS Trust, London SW7 2AZ, UK \\ ${ }^{3}$ Department of Physical Therapy and Rehabilitation Science, Carver College of Medicine, University of Iowa, Iowa City, \\ IA 52242, USA
}

Correspondence should be addressed to Kevin S. Heffernan; ksheffer@syr.edu

Received 14 September 2013; Accepted 25 November 2013; Published 5 January 2014

Academic Editor: Atsunori Kamiya

Copyright (C) 2014 Kevin S. Heffernan et al. This is an open access article distributed under the Creative Commons Attribution License, which permits unrestricted use, distribution, and reproduction in any medium, provided the original work is properly cited.

\begin{abstract}
We examined the peripheral hemodynamic response to passive arm postural changes in young men and women. Radial artery pulse waveforms were captured using applanation tonometry in 20 men (age $27 \pm 2 \mathrm{yrs}$, BMI $25 \pm 1 \mathrm{~kg} / \mathrm{m}^{2}$ ) and 20 women (age $27 \pm 2 \mathrm{yrs}, \mathrm{BMI} 23 \pm 1 \mathrm{~kg} / \mathrm{m}^{2}$ ). Arm position was maintained at either heart level or supported $14 \mathrm{~cm}$ above/below heart level in a randomized fashion. Systolic augmentation index (sAIx) and diastolic augmentation index (dAIx) were used as estimates of pressure from wave reflections arriving in systole and diastole, respectively. A novel reservoir-wave separation technique was used to obtain arterial reservoir pressure (pressure generated by arterial capacitance). Women showed a significant reduction in radial diastolic pressure-time integral (DPTI) $(P<0.05)$ and reservoir pressure $(P<0.05)$, with no change in peripheral sAIx $(P>0.05)$ or $\operatorname{dAIx}(P>0.05)$ when moving the arm from below to above heart level. Conversely, men showed an attenuated change in radial DPTI $(P>0.05)$ concomitant with significant increases in reservoir pressure $(P<0.05)$, sAIx $(P<0.05)$, and dAIx $(P<0.05)$. Gravity-mediated changes in regional hemodynamics produced by passive arm postural shifts are sex specific. Men demonstrate less change in regional diastolic pressure concomitant with increased augmentation index and arterial reservoir pressure.
\end{abstract}

\section{Introduction}

There are well established sex differences in blood pressure (BP) regulation. While hypertension affect more young men, hypotension and subsequent orthostatic intolerance affects more young women [1]. Although numerous mechanisms have been put forth (autonomic, hormonal, etc.), none fully explain sex differences in BP and its regulation [2]. Sex differences in the hemodynamic response to changes in hydrostatic pressure gradients $[3,4]$ have been implicated as an important factor contributing to sex differences in orthostatic tolerance [1]. Gravity is an often neglected factor that contributes to the genesis of pressure in the systemic circulation. According to the hydrostatic theory, liquid in a tube exerts pressure on the vessel wall and this is influenced by the density of the fluid, the height of the column/vertical displacement between two points in the tube, and the acceleration of the fluid medium due to gravitation effects [5]. Recently it has been suggested that hydrostatic effects cannot be the sole arbitrator of pressure changes in the systemic circulation with postural shifts in vivo and additional consideration should be given to dynamic vascular mechanical properties [6].

Pulse waveform analysis may provide novel insight into the passive effects of gravity on vascular-hemodynamic interactions in men and women. Across the human lifespan, BP waveform morphology transitions from one indicative of a wave transmission-reflection model to a more Windkessellike model [7, 8]. According to wave transmission-reflection theory, the BP waveform is an amalgam of forward and backward travelling waves. Although pressure from wave reflections is known to attenuate forward blood flow, it augments incident wave pressure and may thus be involved 
with $\mathrm{BP}$ regulation during a postural perturbation (i.e., preventing a precipitous drop during changes in transmural pressure) [9]. There are well established sex differences in pressure from wave reflections at rest [10] and during perturbation [11]. It is possible that sex differences in pressure from wave reflections $[10,12-14]$ contribute to hemodynamic (dys)regulation during a gravitational perturbation, but this has not been explicitly investigated.

The Windkessel theory holds that as the left ventricle ejects blood into an elastic artery the vessel expands during systole and then recoils during diastole, converting intermittent pulsatile flow to a more laminar smooth flow. Thus the systolic component of the Windkessel ensures optimal input transmission of flow, the diastolic component ensures adequate transfer of flow (i.e., runoff), and both components ensure buffering of excessive pressure and flow pulsatility. Moreover, the Windkessel modulates the interaction of forward and backward travelling waves affecting confluence augmentation $[15,16]$. Sex differences in systemic arterial reservoir pressure remain poorly investigated [17], but potential sex differences with age have been reported with women experiencing larger increases in reservoir pressure across the human lifespan [18]. How mild gravitational perturbations such as limb postural shifts affect regional pressure produced by the arterial Windkessel (i.e., reservoir pressure) in men and women has yet to be explored.

The purpose of the present study was (1) to explore the passive effects of gravity on regional pressure from wave reflections and arterial reservoir pressure in young men and women and (2) to explore potential sex differences in BP wave morphology due to the passive effects of gravity. Systemic perturbations commonly used to explore hemodynamic responsiveness to hydrostatic pressure gradients such as head-up tilt and lower body negative pressure result in robust changes in the neural and hormonal profile $[19,20]$. Given that pressure from wave reflections and arterial reservoir pressure are sensitive to changes in vascular autonomic modulation and heart rate (HR), we employed a mild hydrostatic manipulation (single limb postural change) that has previously been shown to introduce a regional transmural pressure gradient without altering systemic BP, sympathetic activity, $H R$, venoarteriolar reflexes, or vascular resistance [21-26]. This allowed us to perform a detailed examination of a fairly passive and isolated/segmental hemodynamic response devoid of numerous potential confounders of pressure from wave reflections and reservoir pressure. We hypothesized that women would have an attenuated change in pressure from wave reflections and reservoir pressure during mild arm postural shifts compared to age-matched men.

\section{Methods}

2.1. Subjects. Twenty healthy men and twenty healthy women volunteered to participate in this study. Exclusion criteria included self-reported (from a health history questionnaire) hypertension, diabetes mellitus, hyperlipidemia, severe pulmonary disease, neurological disease, and peripheral artery disease. Participants were not taking any medications known to alter cardiovascular (CV) function. Two participants were habitual cigarette smokers (1 male, 1 female). Exclusion of these 2 participants did not alter overall results and therefore they were not excluded from data analyses. For the female participants, there was no standardization for the timing of measurements relative to menstrual cycle phase. Female participants were not taking oral contraceptives. This study was approved by the Institutional Review Board of Syracuse University, and all subjects provided written informed consent before study initiation.

\subsection{Experimental Design. Measures were made in a dimly} lit, temperature controlled room. All subjects refrained from strenuous exercise for $24 \mathrm{~h}$ before testing and all testing was completed in the postprandial state (average $4 \pm 1$ hours) with participants abstaining from caffeine $>6$ hours. Following written consent participants filled out a healthy history questionnaire and a previously validated physical activity questionnaire [27]. This was followed by a brief familiarization period. Height and weight were then measured in all participants using a wall-mounted ruler and electronic scale, respectively.

Participants were allowed to rest in the supine position for 10 minutes prior to study onset. We employed a mild arm postural manipulation that has previously been shown to provoke gravity-mediated changes in regional transmural pressure without altering systemic autonomic status, systemic blood pressure, and heart rate, important confounders of wave reflection and reservoir pressures [21-24, 28]. All measures were made with the participants right arm slightly abducted $\left(\sim 45^{\circ}\right)$. The right wrist was either elevated or lowered $14 \mathrm{~cm}$ above/below heart level and approximated at right atrial level or half the distance between the midsternum and exam table/midaxillary and 4 th intercostal space. This arm positioning theoretically creates a difference in regional perfusion pressure of approximately $21 \mathrm{mmHg}$ [29, 30]. Based on pilot work and previous published suggestions, the arm below displacement height was selected to minimize shoulder deflection and thus maximize participant comfort [21]. The arm above condition was then selected to match this hydrostatic column displacement. The arm and wrist were supported at all times with foam blocks. Participants completed the 3 conditions (below heart level, heart level, and above heart level) in a randomized fashion. In response to arm manipulation there may be dynamic/rapid changes in vascular and hemodynamic variables that occur $1-5 \mathrm{~s}$ after initial change in limb position due to venous emptying followed by a more sustained response/plateau in vascular and hemodynamic properties (30-60 s after change in arm position) $[26,31,32]$. Therefore five minutes were afforded from onset of arm position change to time of radial pulse wave analysis (PWA) measurement in an attempt to capture data during more steady state pressure-flow conditions [21].

2.3. Hemodynamics. Brachial BP was measured with participants in the supine position using an automated oscillometric cuff (Panasonic Ew3109, Secaucus, NJ) [33]. BP measurements were made in duplicate in the right arm 


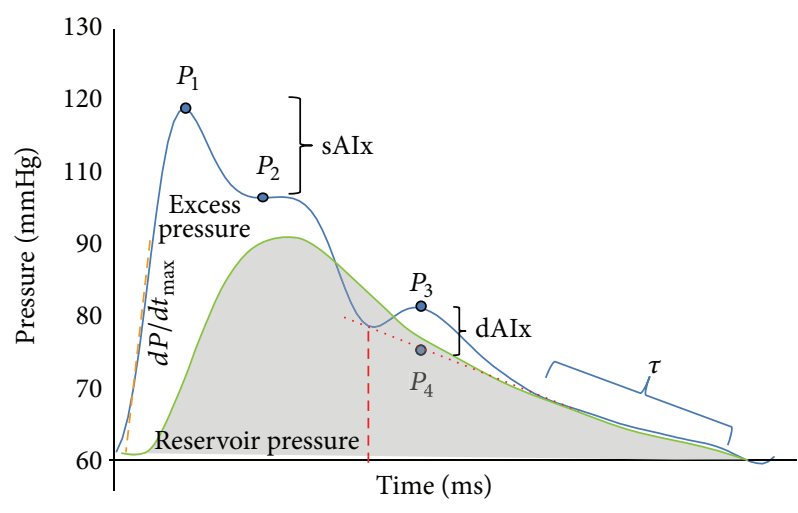

FIGURE 1: Sample ensemble-averaged radial pressure waveform. Systolic augmentation index (sAIx) was calculated as the ratio of amplitude of the late systolic shoulder $\left(P_{2}\right)$ the primary wave pressure peak $\left(P_{1}\right)$ expressed as a percentage $\left(P_{2} / P_{1} * 100\right)$. Diastolic augmentation index (dAIx) was calculated as $\left(P_{3}-P_{4}\right) /(\mathrm{PP}) * 100 . P_{3}$ is the peak diastolic pressure in diastole associated with the dicrotic notch. $P_{4}$ corresponds to a point on a straight line passing through the onset of the incisura of the dicrotic notch and tangential to the natural monoexponential decay of the late diastolic waveform. $d P / d t_{\max }$ was taken as the slope (maximal first derivative) of the maximum rate of pressure rise in systolic pressure per unit time. The slope of the late diastolic decay ( $\tau$, tau) was calculated as the final two-thirds of the diastolic time interval/( $\operatorname{lnESP}-\ln D P)$.

following established guidelines [34]. If these values deviated by more than $5 \mathrm{mmHg}$, additional measurement was conducted and repeated until two consecutive measures were within $5 \mathrm{mmHg}$. Reliability of BP measures in our lab using this approach was assessed in 9 young adults under resting conditions (following overnight fast) on two separate days (average 4 days apart; range 2-7 days apart). The intraclass correlation coefficient was 0.96 .

2.4. Radial Pulse Wave Analysis. Radial artery pressure waveforms were obtained in the right wrist (same arm being posturally manipulated) with participants in the supine position from a 10-second epoch using applanation tonometry (SphygmoCor, AtCor Medical, Sydney, Australia). Radial BP was derived by calibrating the radial pulse wave against brachial mean blood pressure $(\mathrm{MBP})$ and diastolic blood pressure (DBP) given that $\mathrm{MBP}$ and $\mathrm{DBP}$ are consistent throughout the arterial tree. For MBP, a form factor of 0.4 was used as the "one-third" rule may underestimate true MBP [35]. This form factor also more closely conforms to the contour of the radial pulse wave in young adults [36]. Pulse pressure (PP) was calculated as systolic blood pressure (SBP)DBP. Brachial-to-radial PP amplification was calculated as radial $\mathrm{PP} /$ brachial $\mathrm{PP}$.

Systolic augmentation index (sAIx) was calculated as the ratio of amplitude of the late systolic shoulder $\left(P_{2}\right.$, pressure attributable to wave reflections) the primary wave pressure peak $\left(P_{1}\right)$ expressed as a percentage (Figure $\left.1, P_{2} / P_{1} * 100\right)$. Diastolic augmentation index (dAIx) was calculated as $\left(P_{3}-\right.$ $\left.P_{4}\right) /(\mathrm{PP}) * 100[37,38] . P_{3}$ is the peak diastolic pressure in diastole (peak convexity) associated with the dicrotic notch whose amplitude is influenced by diastolic wave reflections
$[37,38] . P_{4}$ corresponds to a point on a straight line passing through the onset of the incisura of the dicrotic notch and tangential to the natural monoexponential decay of the late diastolic waveform that represents the contour of the diastolic waveform devoid of the dicrotic notch/wave reflections [37, 38].

The systolic pressure-time integral (SPTI, the area under the systolic portion of the aortic pressure wave) and the diastolic pressure time index (DPTI, the area under the diastolic portion of the aortic pressure wave) were quantified to examine systolic-to-diastolic shifts in the proportion of pressure comprising the overall pressure waveform. The slope (maximal first derivative) of the maximum rate of pressure rise in systolic pressure per unit time $\left(d P / d t_{\max }\right)$ was taken as a measure of systolic Windkessel vascular coupling given that it is influenced by left ventricular contractility and flow ejection velocity, forward wave pressure (input impedance), and regional compliance. The slope of the radial diastolic decay $(\tau)$ was calculated as the final two-thirds of the diastolic time interval/(lnESP - $\ln \mathrm{DBP})$, where $\operatorname{lnESP}$ is the natural $\log$ of end systolic pressure and $\operatorname{lnDBP}$ is the natural log of diastolic blood pressure, and taken as a crude proxy of diastolic Windkessel vascular coupling given that it is the product of arterial compliance and vascular resistance/terminal impedance [39].

All measurements were made in duplicate, and the mean value was used for subsequent analysis. If device quality index did not exceed 90\%, a third measure was performed and the two highest quality recordings were used for analyses. Reliability of BP waveform morphology in our lab using this technique was assessed in 9 young adults under resting conditions (following overnight fast) on two separate days (average 4 days apart; range 2-7 days apart). Measures were made in duplicate and the average value used for reliability analysis. The intraclass correlation coefficient for peripheral sAIx and dAIx (which encompasses all major pressure components of the radial waveform) was 0.93 and 0.90 , respectively.

In order to further examine the regional Windkessel function, we applied a novel reservoir-wave separation technique to the radial pressure waveform to obtain reservoir pressure (pressure attributable to the capacitance function of the radial artery) and excess wave pressure (pressure attributable nonspecifically to forward and backward travelling waves). When inflow exceeds outflow, the vessel is distended causing a rise in pressure. This arterial reservoir pressure was calculated by separating the aforementioned radial pressure waves into 2 components by iteration of the following equation and by fitting a monoexponential function to the falling pressure during diastole $[15,40]$ :

$$
\begin{aligned}
& P_{\text {reservoir }}-P_{\infty} \\
& \quad=e^{-(a+b) t} \int_{0}^{t}\left[a P\left(t^{\prime}\right)+b P_{\infty}\right] e^{(a+b) t^{\prime}} d t^{\prime}+\left(P_{d}-P_{\infty}\right) e^{-(a+b) t} .
\end{aligned}
$$

The excess wave pressure (pressure attributed to forward and backward travelling waves) was derived by the subtraction of arterial reservoir pressure from total pressure [41]. 
These waveform components provide information that is incremental to systolic and diastolic pressure-time integrals. All of the reservoir and excess wave pressures are quoted with diastolic pressure subtracted. $P_{\text {reservoir }}$ is reservoir pressure, $P_{\infty}$ is the asymptotic pressure, $P_{d}$ is the measured diastolic pressure at $t=0, b=1 / R C$, where $R=$ resistance and $C=$ compliance of the system, and $a$ is a rate constant that can be determined by fitting during the diastolic period. A sample waveform with all hemodynamic variables is presented in Figure 1.

2.5. Statistical Analyses. Baseline sex comparisons for descriptive variables were made with analysis of variance (ANOVA) for continuous variables and chi-square tests for categorical variables. All variables were compared over time using an ANOVA with repeated measures (sex-by-condition comparison). If a significant interaction was detected, post hoc comparisons were made by $t$-test. A Bonferroni adjustment was made for multiple comparisons. Pearson and Spearman correlation coefficients were used to assess relationships between variables of interest. All data are reported as means \pm standard error of the mean (SE). Significance was set $a$ priori as $P<0.05$. All statistical analyses were made using statistical package for the social sciences (IBM SPSS v. 19.0).

\section{Results}

There were no sex differences in age (men $27 \pm 2$ years versus women $27 \pm 2$ years, $P=0.87$ ). Men were significantly taller $(178 \pm 1 \mathrm{~cm}$ versus $165 \pm 1 \mathrm{~cm})$ and had greater body mass $(80 \pm 2 \mathrm{~kg}$ versus $65 \pm 2 \mathrm{~kg})$ than women $(P<0.05)$. There were no significant differences in body mass index (men $25 \pm 1$ versus women $23 \pm 1, P>0.05$ ) or total physical activity levels (men $4742 \pm 490$ versus $4053 \pm 824$ METS-minutes/week, $P>$ 0.05).

Arm manipulation resulted in similar changes across sex in, MBP, brachial and radial SBP, brachial and radial PP and radial SPTI (Tables 1 and 2, $P<0.05$ for time effects; $P>0.05$ for sex-by-condition interactions). Arm manipulation had no effect on PP amplification, $d P / d t_{\text {max }}$, excess wave pressure, ejection duration, or HR (Tables 1 and $2, P>0.05$ for condition effects). Change in the contour of the radial pulse waveform with change in arm position varied considerably by sex (Figure 2).

A significant sex-by-condition interaction was detected for radial DPTI (Table 2, $P<0.05$ ). Women had a lessened increase in radial DPTI with arm below heart level $(P<$ 0.05 ) and a significant reduction in radial DPTI with the arm above heart level $(P<0.05)$. A significant sex-bycondition interaction was detected for both sAIx and dAIx $(P<0.05)$. There was a significant reduction in sAIx $(P<$ $0.05)$ and a nonsignificant reduction in dAIx $(P>0.05)$ with arm below heart level in men. There was a significant increase in sAIx $(P<0.05)$ and a significant increase in dAIx $(P<0.05)$ when moving the arm above heart level in men. There were no significant changes in sAIx or dAIx in women across conditions $(P>0.05)$. A significant sex-by-condition interaction was detected for reservoir pressure (Figure 3, $P<$
0.05). There were slight reductions in reservoir pressure with the arm below heart level in men $(P>0.05)$ with a significant increase with the arm above heart level $(P<0.05)$. There were slight increases in reservoir pressure with the arm below heart level in women $(P>0.05)$ with significant reductions occurring when moving the arm above heart level $(P<0.05)$.

Across conditions when transitioning from below to above heart level, change in DPTI was associated with change in sAIx $(r=0.65, P<0.05)$ and change in dAIx $(r=0.36$, $P<0.05)$ and inversely associated with change in reservoir pressure $(r=-0.42, P<0.05)$ in men only. Change in radial SPTI was inversely associated with change in $d P / d t_{\max }$ $(r=-0.43, P<0.05)$ and change in dAIx $(r=0.34$, $P<0.05)$ in men only. Across conditions when transitioning from below to above heart level change in radial DPTI was inversely associated with change in $\tau(r=-0.64, P<0.05)$ in women only. Change in radial SPTI was inversely associated with change in $\tau(r=-0.44, P<0.05)$ in women only. For both sexes across conditions, change in reservoir pressure was associated with change in sAIx $(r=0.32, P<0.05)$ and change in dAIx $(r=0.37, P<0.05)$.

\section{Discussion}

Gravity is known to have a profound effect on BP. For every centimeter of vertical displacement, it has been estimated that $\mathrm{BP}$ is altered by approximately $0.77 \mathrm{mmHg}$ and this effect is deemed to be predominantly hydrostatic [42]. According to hydrostatic theory, the pressure at any given point in a fluid-containing tube depends on the magnitude of the force imposed on the tube (i.e., gravity), the density of the liquid contained within the tube, and the vertical displacement of the tube relative to "heart level." This theory largely ignores the dynamic nature of arterial mechanical properties [6] and potential contributions of arterial reservoir pressure and pressure from wave reflections to hemodynamic modulation.

The present study set out to explore changes in the radial pulse wave contour with arm position manipulation in order to gain insight into gravity-induced effects on regional $\mathrm{BP}$ in men and women. Our findings suggest that gravity has a disparate effect on regional hemodynamics in men and women. When moving the arm from below to above heart level, men demonstrate an increase in pressure from wave reflections and reservoir pressure. Conversely women experience a reduction in reservoir pressure and no change in pressure from wave reflections. Changes in arterial reservoir pressure and pressure from wave reflections were associated with changes in diastolic pressure in men only. Thus changes in arterial reservoir pressure and pressure from wave reflections may contribute to regional hemodynamic (in)stability when a vessel is exposed to passive hydrostatically mediated pressure gradients and this is sex specific.

Using passive arm manipulation, we noted that men and women had similar changes in forward wave pressure $\left(P_{1}\right.$ height), excess wave pressure, and $d P / d t$ suggesting similar incident pressure wave transmission into the radial artery. However, there were prominent sex differences in pressure from wave reflections arriving during both systole (sAIx) 


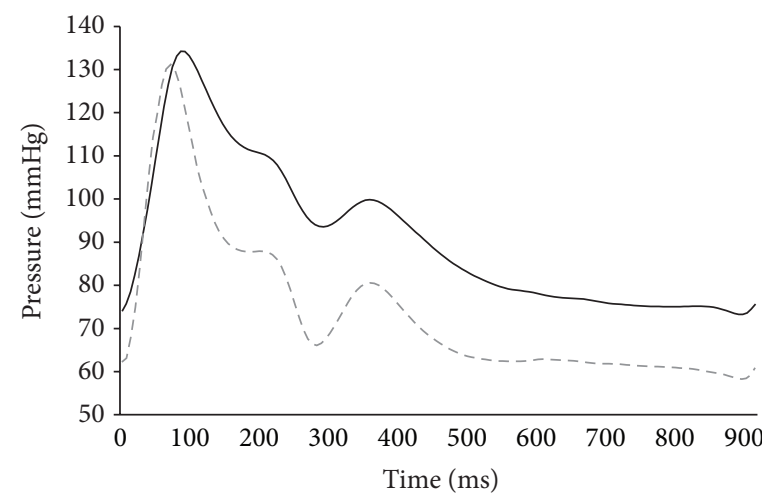

(a)

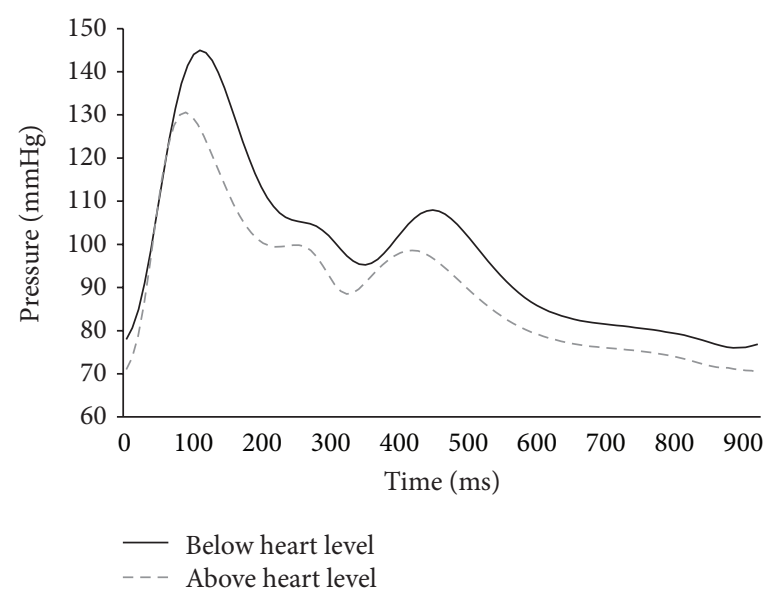

(b)

FIGURE 2: Sample radial pressure waveforms for women (a) and men (b) with the arm supported below heart level and above heart level.

TABLE 1: Brachial and radial blood pressure in men and women.

\begin{tabular}{|c|c|c|c|c|c|}
\hline Variable & Below heart & Neutral & Above heart & Condition effect & Sex-by-condition interaction \\
\hline \multicolumn{6}{|c|}{ Brachial SBP, mmHg } \\
\hline Men & $123 \pm 2$ & $116 \pm 2$ & $111 \pm 2$ & \multirow{2}{*}{$<0.001$} & \multirow{2}{*}{0.840} \\
\hline Women & $115 \pm 2$ & $109 \pm 2$ & $104 \pm 2$ & & \\
\hline \multicolumn{6}{|c|}{ Brachial DBP, mmHg } \\
\hline Men & $76 \pm 1$ & $70 \pm 2$ & $69 \pm 1$ & \multirow{2}{*}{$<0.001$} & \multirow{2}{*}{0.167} \\
\hline Women & $72 \pm 1$ & $69 \pm 2$ & $66 \pm 1$ & & \\
\hline \multicolumn{6}{|c|}{ Brachial PP, mmHg } \\
\hline Men & $47 \pm 2$ & $46 \pm 2$ & $44 \pm 2$ & \multirow{2}{*}{$<0.001$} & \multirow{2}{*}{0.409} \\
\hline Women & $43 \pm 2$ & $40 \pm 2$ & $39 \pm 2$ & & \\
\hline \multicolumn{6}{|c|}{ Radial SBP, mmHg } \\
\hline Men & $144 \pm 3$ & $134 \pm 4$ & $128 \pm 3$ & \multirow{2}{*}{$<0.001$} & \multirow{2}{*}{0.497} \\
\hline Women & $129 \pm 3$ & $123 \pm 4$ & $115 \pm 3$ & & \\
\hline \multicolumn{6}{|c|}{ Radial DBP, mmHg } \\
\hline Men & $76 \pm 1$ & $70 \pm 1$ & $69 \pm 1$ & \multirow{2}{*}{$<0.001$} & \multirow{2}{*}{0.051} \\
\hline Women & $71 \pm 1$ & $69 \pm 1$ & $65 \pm 1$ & & \\
\hline \multicolumn{6}{|c|}{ Radial PP, mmHg } \\
\hline Men & $68 \pm 3$ & $64 \pm 4$ & $59 \pm 4$ & \multirow{2}{*}{$<0.001$} & \multirow{2}{*}{0.702} \\
\hline Women & $57 \pm 3$ & $54 \pm 4$ & $51 \pm 3$ & & \\
\hline \multicolumn{6}{|l|}{ MBP, mmHg } \\
\hline Men & $94 \pm 1$ & $88 \pm 1$ & $86 \pm 1$ & \multirow{2}{*}{$<0.001$} & \multirow{2}{*}{0.173} \\
\hline Women & $89 \pm 1$ & $85 \pm 1$ & $82 \pm 1$ & & \\
\hline \multicolumn{6}{|c|}{ PP amplification } \\
\hline Men & $1.45 \pm 0.04$ & $1.38 \pm 0.05$ & $1.33 \pm 0.06$ & \multirow{2}{*}{0.310} & \multirow{2}{*}{0.258} \\
\hline Women & $1.34 \pm 0.04$ & $1.33 \pm 0.05$ & $1.34 \pm 0.06$ & & \\
\hline
\end{tabular}

Data are mean \pm SEM.

and diastole (dAIx) in men and women when moving the arm from below to above heart level. It is interesting to note that increases in pressure from wave reflections with arm elevation were first reported by Johannes von Kries approximately 120 years ago. von Kries noted that in the normal individual an anacrotic pulse (i.e., pulse waveform with a clear inflection point on the ascending limb) may be obtained by raising the arm [43]. In this position, the reflection of the pulse wave from the periphery was thought to be favored and the "anacrotic wave" simply a quickly reflected wave [43]. In men, change in both sAIx and dAIx was associated with change in regional diastolic pressures. This is significant as men were better able to maintain regional diastolic pressure during arm elevation than women. Thus 
TABLE 2: Hemodynamic parameters derived from the radial pressure waveform in men and women.

\begin{tabular}{|c|c|c|c|c|c|}
\hline Variable & Below heart & Neutral & Above heart & Condition effect & Sex-by-condition interaction \\
\hline \multicolumn{6}{|l|}{ sAIx, $\%$} \\
\hline Men & $39 \pm 3$ & $43 \pm 4$ & $50 \pm 4$ & \multirow{2}{*}{0.001} & \multirow{2}{*}{0.014} \\
\hline Women & $51 \pm 3$ & $52 \pm 4$ & $53 \pm 4$ & & \\
\hline \multicolumn{6}{|l|}{ dAIx, \% } \\
\hline Men & $24 \pm 1$ & $26 \pm 2$ & $31 \pm 2$ & \multirow{2}{*}{0.020} & \multirow{2}{*}{0.038} \\
\hline Women & $22 \pm 1$ & $22 \pm 2$ & $22 \pm 2$ & & \\
\hline \multicolumn{6}{|c|}{$P_{1}$-DBP, mmHg } \\
\hline Men & $68 \pm 3$ & $64 \pm 4$ & $60 \pm 4$ & \multirow{2}{*}{0.006} & \multirow{2}{*}{0.338} \\
\hline Women & $57 \pm 3$ & $54 \pm 4$ & $53 \pm 4$ & & \\
\hline \multicolumn{6}{|l|}{ SPTI, auc } \\
\hline Men & $2202 \pm 75$ & $2080 \pm 71$ & $2024 \pm 66$ & \multirow{2}{*}{$<0.001$} & \multirow{2}{*}{0.387} \\
\hline Women & $2244 \pm 75$ & $2100 \pm 71$ & $2000 \pm 66$ & & \\
\hline \multicolumn{6}{|l|}{ DPTI, auc } \\
\hline Men & $3529 \pm 79$ & $3219 \pm 87$ & $3241 \pm 68$ & \multirow{2}{*}{$<0.001$} & \multirow{2}{*}{0.024} \\
\hline Women & $3172 \pm 79$ & $3033 \pm 87$ & $2742 \pm 68$ & & \\
\hline \multicolumn{6}{|c|}{$d P / d t_{\max }, \mathrm{mmHg} \mathrm{s}^{-1}$} \\
\hline Men & $1079 \pm 61$ & $1043 \pm 71$ & $979 \pm 75$ & \multirow{2}{*}{0.596} & \multirow{2}{*}{0.282} \\
\hline Women & $883 \pm 61$ & $872 \pm 77$ & $908 \pm 76$ & & \\
\hline \multicolumn{6}{|c|}{ Excess pressure, mmHg } \\
\hline Men & $39 \pm 2$ & $37 \pm 3$ & $35 \pm 3$ & \multirow{2}{*}{0.109} & \multirow{2}{*}{0.680} \\
\hline Women & $30 \pm 2$ & $29 \pm 3$ & $29 \pm 3$ & & \\
\hline \multicolumn{6}{|c|}{$\tau, \mathrm{mmHg} \mathrm{ms}^{-1}$} \\
\hline Men & $187 \pm 12$ & $172 \pm 9$ & $161 \pm 15$ & \multirow{2}{*}{0.358} & \multirow{2}{*}{0.048} \\
\hline Women & $144 \pm 12$ & $157 \pm 9$ & $184 \pm 14$ & & \\
\hline \multicolumn{6}{|c|}{ Ejection duration, ms } \\
\hline Men & $321 \pm 5$ & $323 \pm 5$ & $323 \pm 5$ & \multirow{2}{*}{0.481} & \multirow{2}{*}{0.759} \\
\hline Women & $340 \pm 5$ & $342 \pm 5$ & $338 \pm 5$ & & \\
\hline \multicolumn{6}{|c|}{ Heart rate, bpm } \\
\hline Men & $63 \pm 3$ & $63 \pm 2$ & $62 \pm 2$ & \multirow{2}{*}{0.294} & \multirow{2}{*}{0.576} \\
\hline Women & $64 \pm 3$ & $63 \pm 2$ & $63 \pm 2$ & & \\
\hline
\end{tabular}

Data are mean \pm SEM.

gravity-induced changes in pressure from wave reflections may contribute to regional hemodynamic modulation during passive upper limb postural shifts.

There were prominent sex differences in regional arterial reservoir pressure during arm postural shifts. Unlike what was seen in women, men experienced an increase in arterial reservoir pressure when moving the arm from below to above heart level and this was associated with regional changes in diastolic pressure. When arterial inflow into a vessel exceeds outflow capacity, there is an increase in volume which distends the vessel causing radial expansion. This distension generates pressure (i.e., reservoir pressure) [15]. Thus reservoir pressure is proportional to the instantaneous difference between input and terminal impedance and transverse impedance/arterial compliance [15]. Our findings suggest that this regional arterial reservoir is affected by gravitational effects and may contribute to localized pressure regulation in men and women produced by passive arm postural shifts. Men generate more pressure from the arterial reservoir during reductions in transmural pressure and this is associated with preservation of diastolic pressures.

Previous work from Davies et al. has noted that AIx is strongly influenced by the arterial reservoir [15]. This is consistent with present findings as we noted an association between change in reservoir pressure and change in sAIx and dAIx in both sexes. While pulse wave velocity and heart rate affect timing of wave reflection arrival, the arterial reservoir may influence wave reflection magnitude and subsequent augmentation [16]. The arterial reservoir may be an important modulator of sex differences in the hemodynamic response to hydrostatically mediated changes in transmural pressure. Reductions in regional blood pressure in women in response to limb postural shifts may be mediated by disparate changes in the arterial reservoir coupled with blunted pressure modulation from wave reflections.

The majority of reservoir pressure in the systemic circulation is expected to be generated by large elastic central arteries. However, a small regional effect may exist. 


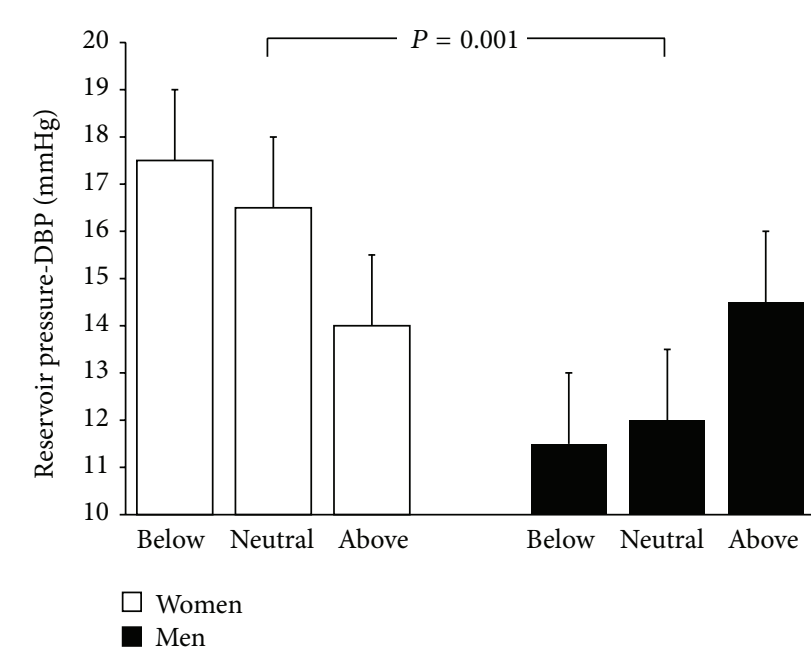

FIGURE 3: Changes in reservoir pressure with arm manipulation in women (white bars) and men (black bars). A significant sex-bycondition interaction was detected $(P=0.001)$.

Reservoir pressure obtained from invasive and noninvasive pressure recordings of the aorta/carotid has been estimated to be approximately $25-45 \mathrm{mmHg}$ (less DBP) in younger middle aged adults $[15,18,44]$. We noted resting values of approximately $14 \mathrm{mmHg}$ obtained from radial pressure waveforms suggesting differences between central elastic and peripheral muscular arteries. Moreover, changes in reservoir pressure with arm manipulation were small $(\sim 2-3 \mathrm{mmHg})$. It is unlikely that subtle arm manipulation produced systemic changes in large artery function and central reservoir pressure. Indeed, when manipulating height of the left arm while capturing radial pressure waves in the right wrist at heart level in a subset of participants $(n=4)$, we noted no change in BP in the right brachial artery and no change in the contour of the right radial pressure wave (no change in any parameter measured). Thus we believe our findings to suggest the presence of a small regional reservoir effect. However small, it may still affect the contour of the regional pressure wave (Figure 1) and thus segmental hemodynamics.

The resting caliber of an artery depends on the balance between passive (pressure-induced) expansion and active contraction/wall tension. In response to changes in hydrostatic pressure with passive changes in arm position, this balance is influenced by complex changes in vascular tone, compliance, elastic modulus, viscoelasticity, and stress-strain (i.e., hysteresis and vascular creep) $[45,46]$. With the arm above heart level, women experienced an increase in $\tau$, a term that is heavily influenced by arterial compliance. Change in $\tau$ was associated with change in diastolic pressure in women only. Increases in arterial compliance would be expected to result in reductions in reservoir pressure and pressure from wave reflections. Thus gravity-mediated changes in arterial compliance could contribute to sex differences in peripheral hemodynamics in vivo. There may also be sex differences in the physical composition of the radial artery [47] and this altered matrix combined with differential interactions of elastin, collagen, and smooth muscle could influence hemodynamic reactivity to postural limb shifts.
Sex differences in the vascular and hemodynamic response to changes in hydrostatic pressure gradients produced via systemic orthostatic challenges have been well documented $[3,4]$ and this attenuated local autoregulatory vascular responsiveness has been implicated in predisposition to orthostatic hypotension in women $[3,4]$. In response to gross systemic perturbations such as standing, head-up tilt, or lower body negative pressure, young women demonstrate a disproportionate propensity toward inability to maintain BP $[22,48]$. During head-up tilt, there is a generalized increase in systemic DBP in healthy young men [49]. Conversely, it has been noted that change in DBP during upright tilt in women is only $29 \%$ of that seen in men [49]. In response to changes in transmural pressure with lower body negative pressure (LBNP), women have a significantly greater reduction in DBP compared to men [3]. Changes in pressure from wave reflections and reservoir pressure during postural shifts have logically been implicated in the BP response to these maneuvers but not specifically examined $[9,20,37,50]$.

During head-up tilt, there are reductions in wave reflection magnitude despite increases in peripheral vascular resistance $[20,50]$. This finding has been recapitulated using LBNP [19]. In men, increases in muscle sympathetic nerve activity (MSNA) are associated with increases in total peripheral resistance (TPR) [51], increases in pressure from wave reflections [52], and greater coherence with oscillations in DBP [53]. Conversely, in women, increases in MSNA are not associated with TPR [51], inversely associated with pressure from wave reflections [52], and weakly associated with oscillations in DBP [53]. Our findings build upon these observations and suggest a sex-specific role for wave reflections in affecting regional hemodynamics during passive limb postural shifts. Inability to maintain BP in response to changes in transmural pressure in women may be partially related to inability to augment pressure from wave reflections. More research will be needed to explore sex differences in arterial reservoir pressure and wave reflections in response to postural shifts.

Limitations to this study should be noted. We did not control for menstrual phase. After accounting for change in heart rate, menstrual cycle does not appear to influence the contour of the pulse waveform [54-56], although this is not a universal finding [57]. Although menstrual phase may influence autonomic neural control of BP and its regulation/reactivity, the current arm position manipulation was selected as it produces modest and passive regional vascular/hemodynamic effects independent of hormonal modulation and sympathetic nerve traffic. Therefore, we do not believe that the passive hemodynamic effects witnessed herein are confounded by hormonal influences secondary to the menstrual cycle. Additional research is needed to explore the effect of menstrual cycle on BP response to changes in arm position.

In conclusion, passive gravitational effects produce disparate changes in arterial reservoir pressure and pressure from wave reflections in men and women and these changes are associated with regional BP. With reductions in transmural pressure from arm elevation, men are better able to preserve diastolic pressure and this may be related to 
increased arterial reservoir pressure and pressure from wave reflections. Women are less successful maintaining regional diastolic pressure and this may be due to reductions in arterial reservoir pressure coupled with inability to increase pressure from wave reflections. Future research is needed to examine the contribution of these novel hemodynamic indices to BP regulation during systemic orthostatic challenge.

\section{Conflict of Interests}

The authors have no conflict of interests to disclose.

\section{Acknowledgment}

This study was funded in part by support from the Joan N. Burstyn Endowed Fund for Collaborative Research in Education at Syracuse University.

\section{References}

[1] Y. S. Ali, N. Daamen, G. Jacob et al., "Orthostatic intolerance: a disorder of young women," Obstetrical and Gynecological Survey, vol. 55, no. 4, pp. 251-259, 2000.

[2] W. D. Franke, C. P. Johnson, J. A. Steinkamp, R. Wang, and J. R. Halliwill, "Cardiovascular and autonomic responses to lower body negative pressure: do not explain gender differences in orthostatic tolerance," Clinical Autonomic Research, vol. 13, no. 1, pp. 36-44, 2003.

[3] M. E. J. Lott, C. Hogeman, M. Herr, M. Bhagat, and L. I. Sinoway, "Sex differences in limb vasoconstriction responses to increases in transmural pressures," The American Journal of PhysiologyHeart and Circulatory Physiology, vol. 296, pp. H186-H194, 2009.

[4] J. K. Shoemaker, R. L. Hughson, and L. I. Sinoway, "Gender affects sympathetic neurovascular control during postural stress," Journal of Gravitational Physiology, vol. 9, no. 1, pp. P83P84, 2002.

[5] H. Hinghofer-Szalkay, "Gravity, the hydrostatic indifference concept and the cardiovascular system," European Journal of Applied Physiology, vol. 111, no. 2, pp. 163-174, 2011.

[6] B. Gavish and L. Gavish, "Blood pressure variation in response to changing arm cuff height cannot be explained solely by the hydrostatic effect," Journal of Hypertension, vol. 29, no. 11, pp. 2099-2104, 2011.

[7] P. Segers, E. R. Rietzschel, M. L. de Buyzere et al., "Noninvasive (input) impedance, pulse wave velocity, and wave reflection in healthy middle-aged men and women," Hypertension, vol. 49, no. 6, pp. 1248-1255, 2007.

[8] M. W. Mohiuddin, G. A. Laine, and C. M. Quick, "Increase in pulse wavelength causes the systemic arterial tree to degenerate into a classical windkessel," The American Journal of Physiology-Heart and Circulatory Physiology, vol. 293, no. 2, pp. H1164H1171, 2007.

[9] Y. Tabara, J. Nakura, I. Kondo, T. Miki, and K. Kohara, "Orthostatic systolic hypotension and the reflection pressure wave," Hypertension Research, vol. 28, no. 6, pp. 537-543, 2005.

[10] C. D. Gatzka, B. A. Kingwell, J. D. Cameron et al., "Gender differences in the timing of arterial wave reflection beyond diff- erences in body height," Journal of Hypertension, vol. 19, no. 12, pp. 2197-2203, 2001.

[11] D. P. Casey, T. B. Curry, M. J. Joyner, N. Charkoudian, and E. C. Hart, "Acute $\beta$-adrenergic blockade increases aortic wave reflection in young men and women: differing mechanisms between sexes," Hypertension, vol. 59, no. 1, pp. 145-150, 2012.

[12] C. S. Hayward and R. P. Kelly, "Gender-related differences in the central arterial pressure waveform," Journal of the American College of Cardiology, vol. 30, no. 7, pp. 1863-1871, 1997.

[13] C. Y. Shim, S. Park, D. Choi et al., "Sex differences in central hemodynamics and their relationship to left ventricular diastolic function," Journal of the American College of Cardiology, vol. 57, no. 10, pp. 1226-1233, 2011.

[14] C. Russo, Z. Jin, V. Palmieri et al., "Arterial stiffness and wave reflection: sex differences and relationship with left ventricular diastolic function," Hypertension, vol. 60, pp. 362-368, 2012.

[15] J. E. Davies, J. Baksi, D. P. Francis et al., "The arterial reservoir pressure increases with aging and is the major determinant of the aortic augmentation index," The American Journal of Physiology-Heart and Circulatory Physiology, vol. 298, no. 2, pp. H580-H586, 2010.

[16] E. Laffon, C. Galy-Lacour, F. Laurent, D. Ducassou, and R. Marthan, "MRI quantification of the role of the reflected pressure wave on coronary and ascending aortic blood flow," Physiological Measurement, vol. 24, no. 3, pp. 681-692, 2003.

[17] L. Cymberknop, D. Bia, Y. Zocalo et al., "Gender-related differences in the excess pressure component of central aortic pressure waveform of healthy young," in Proceedings of the Annual International Conference of the IEEE Engineering in Medicine and Biology Society (EMBS '11), pp. 207-210, Boston, Mass, USA, September 2011.

[18] S. J. Vermeersch, E. R. Rietzschel, M. L. de Buyzere et al., “The reservoir pressure concept: the 3-element windkessel model revisited? Application to the Asklepios population study," Journal of Engineering Mathematics, vol. 64, no. 4, pp. 417-428, 2009.

[19] C. Lydakis, A. Momen, C. Blaha, M. Herr, U. A. Leuenberger, and L. I. Sinoway, "Changes of elastic properties of central arteries during acute static exercise and lower body negative pressure," European Journal of Applied Physiology, vol. 102, no. 6, pp. 633-641, 2008.

[20] A. M. T. Huijben, F. U. S. Mattace-Raso, J. Deinum, J. Lenders, and A. H. van den Meiracker, "Aortic augmentation index and pulse wave velocity in response to head-up tilting: effect of autonomic failure," Journal of Hypertension, vol. 30, no. 2, pp. 307$314,2012$.

[21] M. F. Frances, R. Goswami, M. Rachinsky et al., "Adrenergic and myogenic regulation of viscoelasticity in the vascular bed of the human forearm," Experimental Physiology, vol. 96, no. 11, pp. 1129-1137, 2011.

[22] S. S. Jarvis, J. P. Florian, M. J. Curren, and J. A. Pawelczyk, "Sex differences in vasoconstrictor reserve during 70 deg head-up tilt," Experimental Physiology, vol. 95, no. 1, pp. 184-193, 2010.

[23] D. Michikami, A. Kamiya, F. Qi, Y. Niimi, S. Iwase, and T. Mano, "Arm elevation enhances muscle sympathetic nerve activity during static exercise," Environmental Medicine, vol. 44, no. 1, pp. 46-48, 2000.

[24] D. Michikami, A. Kamiya, Q. Fu et al., "Forearm elevation augments sympathetic activation during handgrip exercise in humans," Clinical Science, vol. 103, no. 3, pp. 295-301, 2002. 
[25] M. Zamir, R. Goswami, D. Salzer, and J. K. Shoemaker, "Role of vascular bed compliance in vasomotor control in human skeletal muscle," Experimental Physiology, vol. 92, no. 5, pp. 841-848, 2007.

[26] M. E. Tschakovsky and R. L. Hughson, "Venous emptying mediates a transient vasodilation in the human forearm," The American Journal of Physiology-Heart and Circulatory Physiology, vol. 279, no. 3, pp. H1007-H1014, 2000.

[27] C. L. Craig, A. L. Marshall, M. Sjöström et al., "International physical activity questionnaire: 12 -country reliability and validity," Medicine and Science in Sports and Exercise, vol. 35, no. 8, pp. 1381-1395, 2003.

[28] P. Beaconsfield and J. Ginsburg, "Effect of changes in limb posture on peripheral blood flow," Circulation Research, vol. 3, pp. 478-482, 1955.

[29] R. T. Netea, J. W. M. Lenders, P. Smits, and T. Thien, "Both body and arm position significantly influence blood pressure measurement," Journal of Human Hypertension, vol. 17, no. 7, pp. 459-462, 2003.

[30] R. T. Netea, P. J. Bijlstra, J. W. M. Lenders, P. Smits, and T. Thien, "Influence of the arm position on intra-arterial blood pressure measurement," Journal of Human Hypertension, vol. 12, no. 3, pp. 157-160, 1998.

[31] M. E. Lott, M. D. Herr, and L. I. Sinoway, "Effects of age on brachial artery myogenic responses in humans," The American Journal of Physiology-Regulatory Integrative and Comparative Physiology, vol. 287, no. 3, pp. R586-R591, 2004.

[32] M. E. Lott, M. D. Herr, and L. I. Sinoway, "Effects of transmural pressure on brachial artery mean blood velocity dynamics in humans," The Journal of Applied Physiology, vol. 93, no. 6, pp. 2137-2146, 2002.

[33] E. Bonso, F. Dorigatti, and P. Palatini, "Validation of Panasonic EW3106 and EW3109 devices for blood pressure measurement according to the International Protocol," Blood Pressure Monitoring, vol. 15, no. 1, pp. 55-58, 2010.

[34] T. G. Pickering, J. E. Hall, L. J. Appel et al., "Recommendations for blood pressure measurement in humans: an AHA scientific statement from the Council on High Blood Pressure Research Professional and Public Education Subcommittee," Journal of Clinical Hypertension, vol. 7, no. 2, pp. 102-109, 2005.

[35] D. Mahieu, J. Kips, E. R. Rietzschel et al., "Noninvasive assessment of central and peripheral arterial pressure (waveforms): implications of calibration methods," Journal of Hypertension, vol. 28, no. 2, pp. 300-305, 2010.

[36] P. Segers, D. Mahieu, J. Kips et al., "Amplification of the pressure pulse in the upper limb in healthy, middle-aged men and women," Hypertension, vol. 54, no. 2, pp. 414-420, 2009.

[37] L. Jaccoud, C. Rotaru, A. Heim et al., "Major impact of body position on arterial stiffness indices derived from radial applanation tonometry in pregnant and nonpregnant women," Journal of Hypertension, vol. 30, pp. 1161-1168, 2012.

[38] A. Heim, L. Liaudet, B. Waeber, and F. Feihl, "Pulse wave analysis of aortic pressure: diastole should also be considered," Journal of Hypertension, vol. 31, no. 1, pp. 94-102, 2012.

[39] N. Stergiopulos, J.-J. Meister, and N. Westerhof, "Evaluation of methods for estimation of total arterial compliance," The American Journal of Physiology-Heart and Circulatory Physiology, vol. 268, no. 4, pp. H1540-H1548, 1995.
[40] J. E. Sharman, J. E. Davies, C. Jenkins, and T. H. Marwick, "Augmentation index, left ventricular contractility, and wave reflection," Hypertension, vol. 54, no. 5, pp. 1099-1105, 2009.

[41] J. Aguado-Sierra, J. Alastruey, J.-J. Wang, N. Hadjiloizou, J. Davies, and K. H. Parker, "Separation of the reservoir and wave pressure and velocity from measurements at an arbitrary location in arteries," Journal of Engineering in Medicine, vol. 222, no. 4, pp. 403-416, 2008.

[42] P. L. Mitchell, R. W. Parlin, and H. blackburn, "Effect of vertical displacement of the arm on indirect blood-pressure measurement," The New England Journal of Medicine, vol. 271, pp. 72$74,1964$.

[43] W. H. Howell, Textbook of Physiology, W. B. Saunders, London, UK, 1906.

[44] M. G. Schultz, J. E. Davies, P. Roberts-Thomson, J. A. Black, A. D. Hughes, and J. E. Sharman, "Exercise central (aortic) blood pressure is predominantly driven by forward traveling waves, not wave reflection," Hypertension, vol. 62, pp. 175-182, 2013.

[45] A. J. Bank, H. Wang, J. E. Holte, K. Mullen, R. Shammas, and S. H. Kubo, "Contribution of collagen, elastin, and smooth muscle to in vivo human brachial artery wall stress and elastic modulus," Circulation, vol. 94, no. 12, pp. 3263-3270, 1996.

[46] A. J. Bank and D. R. Kaiser, "Smooth muscle relaxation: effects on arterial compliance, distensibility, elastic modulus, and pulse wave velocity," Hypertension, vol. 32, no. 2, pp. 356-359, 1998.

[47] W. Osika, F. Dangardt, S. M. Montgomery, R. Volkmann, L. M. Gan, and P. Friberg, "Sex differences in peripheral artery intima, media and intima media thickness in children and adolescents," Atherosclerosis, vol. 203, no. 1, pp. 172-177, 2009.

[48] J. K. Shoemaker, C. S. Hogeman, M. Khan, D. S. Kimmerly, and L. I. Sinoway, "Gender affects sympathetic and hemodynamic response to postural stress," The American Journal of Physiology-Heart and Circulatory Physiology, vol. 281, no. 5, pp. H2028-H2035, 2001.

[49] R. Schondorf and P. A. Low, "Gender related differences in the cardiovascular responses to upright tilt in normal subjects," Clinical Autonomic Research, vol. 2, no. 3, pp. 183-187, 1992.

[50] A. Tahvanainen, M. Leskinen, J. Koskela et al., "Ageing and cardiovascular responses to head-up tilt in healthy subjects," Atherosclerosis, vol. 207, no. 2, pp. 445-451, 2009.

[51] E. C. Hart, N. Charkoudian, B. G. Wallin, T. B. Curry, J. H. Eisenach, and M. J. Joyner, "Sex differences in sympathetic neuralhemodynamic balance implications for human blood pressure regulation," Hypertension, vol. 53, no. 3, pp. 571-576, 2009.

[52] D. P. Casey, T. B. Curry, M. J. Joyner, N. Charkoudian, and E. C. Hart, "Relationship between muscle sympathetic nerve activity and aortic wave reflection characteristics in young men and women," Hypertension, vol. 57, no. 3, pp. 421-427, 2011.

[53] H. Yang, W. H. Cooke, K. S. Reed, and J. R. Carter, "Sex differences in hemodynamic and sympathetic neural firing patterns during orthostatic challenge in humans," The Journal of Applied Physiology, vol. 112, pp. 1744-1751, 2012.

[54] T. G. Papaioannou, K. S. Stamatelopoulos, G. Georgiopoulos et al., "Arterial wave reflections during the menstrual cycle of healthy women: a reproducibility study, Hypertension, vol. 54, no. 5, pp. 1021-1027, 2009.

[55] A. O. Robb, N. L. Mills, J. N. Din et al., "Influence of the menstrual cycle, pregnancy, and preeclampsia on arterial stiffness," $\mathrm{Hy}$ pertension, vol. 53, no. 6, pp. 952-958, 2009. 
[56] K. S. Stamatelopoulos, G. Georgiopoulos, T. Papaioannou et al., "Can premenstrual syndrome affect arterial stiffness or blood pressure?” Atherosclerosis, vol. 224, no. 1, pp. 170-176, 2012.

[57] E. J. Adkisson, D. P. Casey, D. T. Beck, A. N. Gurovich, J. S. Martin, and R. W. Braith, "Central, peripheral and resistance arterial reactivity: fluctuates during the phases of the menstrual cycle," Experimental Biology and Medicine, vol. 235, no. 1, pp. 111-118, 2010. 

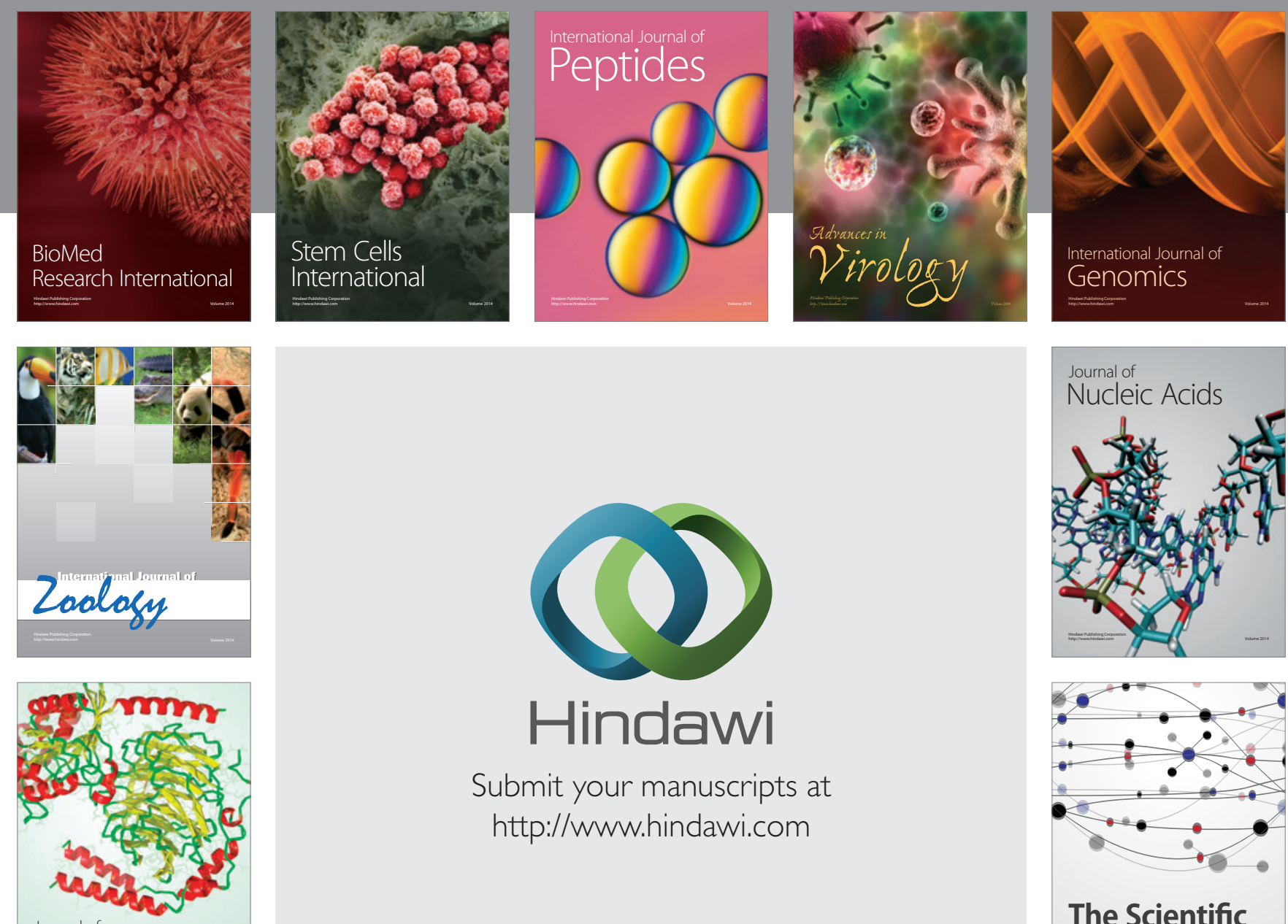

Submit your manuscripts at

http://www.hindawi.com

Journal of
Signal Transduction
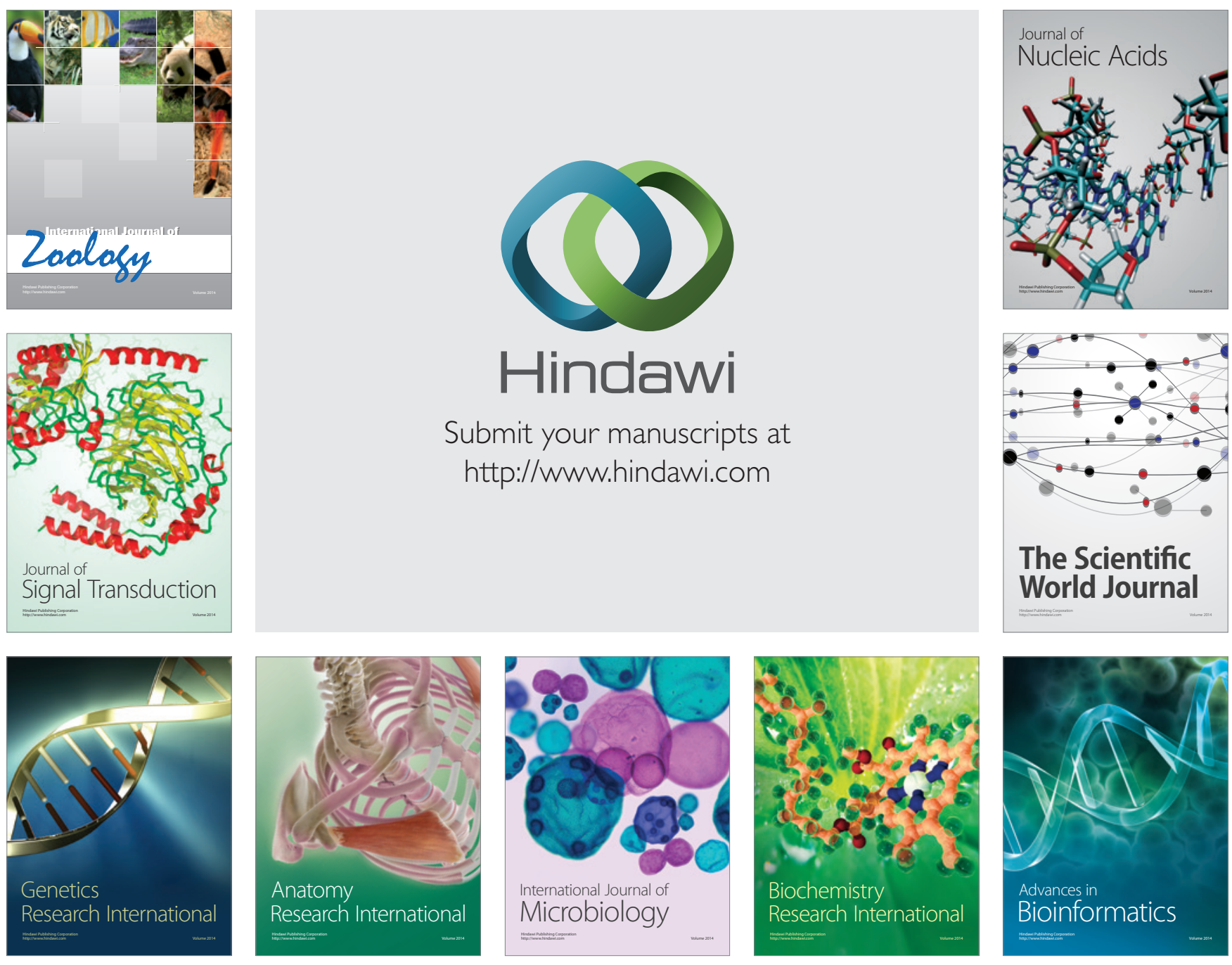

The Scientific World Journal
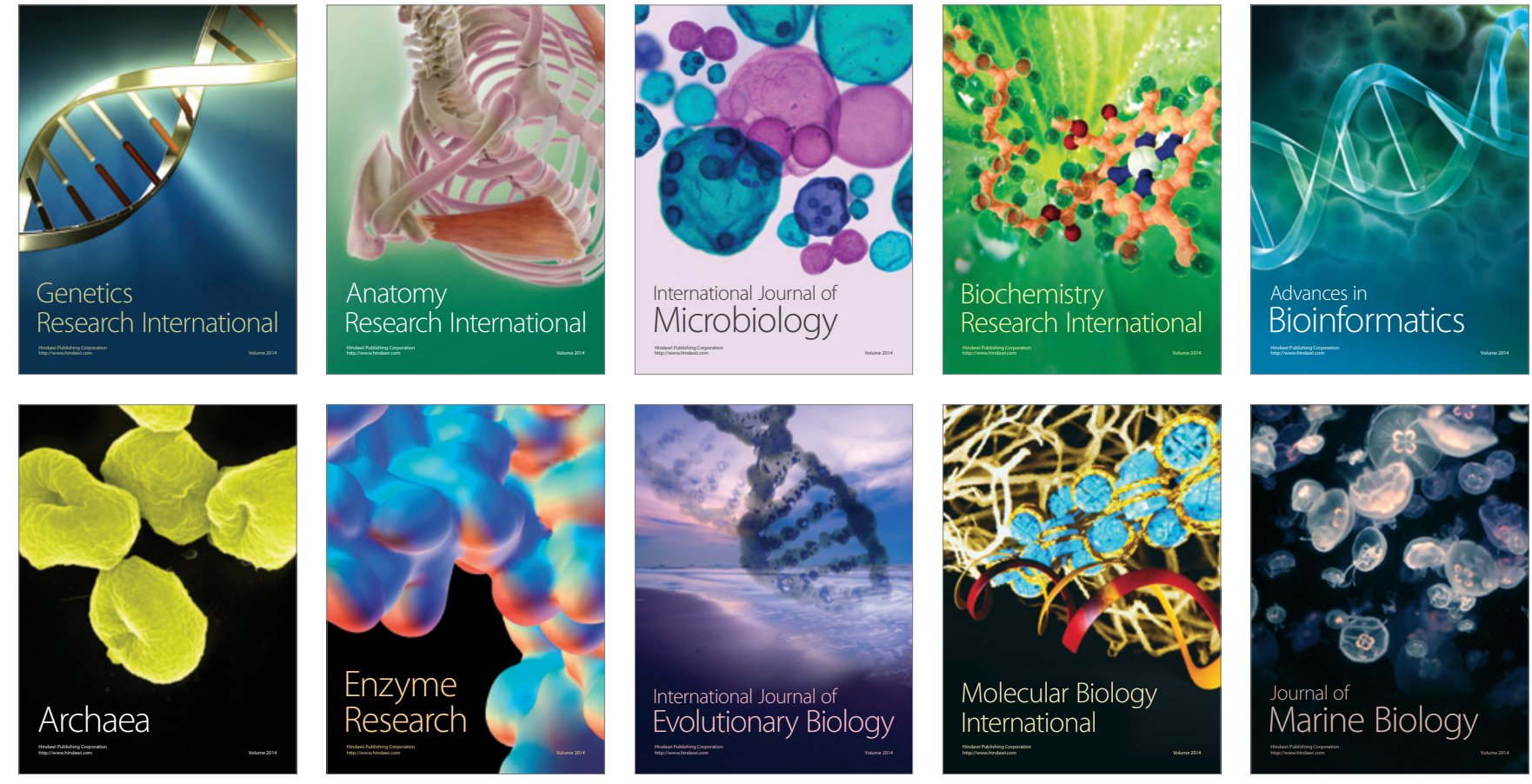